PROCEEDINGS OF THE

AMERICAN MATHEMATICAL SOCIETY

Volume 137, Number 2, February 2009, Pages 641-651

S 0002-9939(08)09609-3

Article electronically published on September 4, 2008

\title{
THE ESCAPING SET OF A QUASIREGULAR MAPPING
}

\author{
WALTER BERGWEILER, ALASTAIR FLETCHER, JIM LANGLEY, AND JANIS MEYER
}

(Communicated by Mario Bonk)

\begin{abstract}
We show that if the maximum modulus of a quasiregular mapping $f: \mathbb{R}^{N} \rightarrow \mathbb{R}^{N}$ grows sufficiently rapidly, then there exists a nonempty escaping set $I(f)$ consisting of points whose forward orbits under iteration of $f$ tend to infinity. We also construct a quasiregular mapping for which the closure of $I(f)$ has a bounded component. This stands in contrast to the situation for entire functions in the complex plane, for which all components of the closure of $I(f)$ are unbounded and where it is in fact conjectured that all components of $I(f)$ are unbounded.
\end{abstract}

\section{INTRODUCTION}

In the study [1] of the dynamics of nonlinear entire functions $f: \mathbb{C} \rightarrow \mathbb{C}$ considerable recent attention has focussed on the escaping set

$$
I(f)=\left\{z \in \mathbb{C}: \lim _{n \rightarrow \infty} f^{n}(z)=\infty\right\},
$$

where $f^{1}=f, f^{n+1}=f \circ f^{n}$ denote the iterates of $f$. Eremenko [5] proved that if $f$ is transcendental, then $I(f) \neq \emptyset$ and indeed that, in keeping with the nonlinear polynomial case [18, the boundary of $I(f)$ is the Julia set $J(f)$. The proof in 5 ] that $I(f)$ is nonempty is based on the Wiman-Valiron theory [6].

For transcendental entire functions $f$, Eremenko went on to prove in [5] that all components of the closure of $I(f)$ are unbounded, and to conjecture that the same is true of $I(f)$ itself. For entire functions with bounded postcritical set this conjecture was proved by Rempe [12, and for the general case it was shown by Rippon and Stallard [16] that $I(f)$ has at least one unbounded component.

In the meromorphic case the set $I(f)$ was first studied by Dominguez [4, who proved that again $I(f) \neq \emptyset$ and $\partial I(f)=J(f)$. For meromorphic $f$ it is possible that all components of $I(f)$ are bounded [4, and the closure of $I(f)$ may have bounded components even if $f$ has only one pole [4, p. 229]. On the other hand $I(f)$ always has at least one unbounded component if the inverse function $f^{-1}$ has a direct transcendental singularity over infinity: this was proved by Bergweiler, Rippon and Stallard [3] by developing an analogue of the Wiman-Valiron theory in the presence of a direct singularity.

Received by the editors February 7, 2008.

2000 Mathematics Subject Classification. Primary 30C65, 30C62; Secondary 37F10.

This research was supported by the G.I.F, the German-Israeli Foundation for Scientific Research and Development, Grant G-809-234.6/2003, and the EU Research Training Network CODY (first author); EPSRC grant RA22AP (second and third authors); the ESF Research Networking Programme HCAA (first and third authors); and DFG grant ME 3198/1-1 (fourth author).

(C)2008 American Mathematical Society Reverts to public domain 28 years from publication 
The present paper is concerned with the escaping set for quasiregular mappings $f: \mathbb{R}^{N} \rightarrow \mathbb{R}^{N}[15$, which represent a natural counterpart in higher real dimensions of analytic functions in the plane, and exhibit many analogous properties, a highlight among these being Rickman's Picard theorem for entire quasiregular maps [13, 15. For the precise definition and further properties of quasiregular mappings we refer the reader to Rickman's text [15]. Now the iterates of an entire quasiregular map are again quasiregular, and properties such as the existence of periodic points were investigated in [2, 17]. Further, there is increasing interest in the dynamics of quasiregular mappings on the compactification $\overline{\mathbb{R}^{N}}$ of $\mathbb{R}^{N}$, although attention has been restricted to mappings which are uniformly quasiregular in the sense that all iterates have a common bound on their dilatation; see [8, Section 21] and [7]. In the absence of this uniform quasiregularity there are evidently some difficulties in extending some concepts of complex dynamics to quasiregular mappings in general, but the escaping set $I(f)$ makes sense nevertheless, and we shall prove the following theorem.

Theorem 1.1. Let $N \geq 2$ and $K>1$. Then there exists $J>1$, depending only on $N$ and $K$, with the following property.

Let $R>0$ and let $f: D_{R} \rightarrow \mathbb{R}^{N}$ be a $K$-quasiregular mapping, where $D_{R} \subseteq \mathbb{R}^{N}$ is a domain containing the set

$$
B_{R}=\left\{x \in \mathbb{R}^{N}: R \leq|x|<\infty\right\} .
$$

Assume that $f$ satisfies

$$
\liminf _{r \rightarrow \infty} \frac{M(r, f)}{r} \geq J, \quad \text { where } M(r, f)=\max \{|f(x)|:|x|=r\},
$$

and define the escaping set by

$$
I(f)=\left\{x \in \mathbb{R}^{N}: \lim _{n \rightarrow \infty} f^{n}(x)=\infty\right\}, \quad f^{1}=f, \quad f^{n+1}=f \circ f^{n} .
$$

Then $I(f)$ is nonempty. If, in addition, $f$ is $K$-quasiregular on $\mathbb{R}^{N}$, then $I(f)$ has an unbounded component.

The proof of Theorem 1.1] is based on the approach of Dominguez [4, as well as that of Rippon and Stallard [16]. A key role is played also by the analogue of Zalcman's lemma [19, 20] developed for quasiregular mappings by Miniowitz 10] (see 92 ). It seems worth observing that in Theorem 1.1 the hypothesis (2) cannot be replaced by

$$
\liminf _{r \rightarrow \infty} \frac{M(r, f)}{r}>1
$$

as the following example shows. Take cylindrical polar coordinates $r \cos \theta, r \sin \theta, x_{3}$ in $\mathbb{R}^{3}$, let $\lambda>0$ and let $f$ be the mapping defined by

$$
0 \rightarrow 0, \quad\left(r e^{i \theta}, x_{3}\right) \rightarrow\left(r e^{\lambda \cos \theta+i(\theta+\pi)}, x_{3}\right) .
$$

Then $f^{2}$ is given by

$$
\left(r e^{i \theta}, x_{3}\right) \rightarrow\left(r e^{\lambda \cos \theta+\lambda \cos (\theta+\pi)+i(\theta+2 \pi)}, x_{3}\right)
$$

and so is the identity, while since $f$ is $C^{1}$ on $\mathbb{R}^{3} \backslash\{0\}$ and satisfies $f(2 x)=2 f(x)$ it is easy to see that $f$ is quasiconformal on $\mathbb{R}^{3}$. On the other hand if $f: \mathbb{R}^{N} \rightarrow \mathbb{R}^{N}$ is quasiregular with an essential singularity at infinity, then $M(r, f) / r \rightarrow \infty$ as $r \rightarrow \infty$ (see, for example, [2, Lemma 3.4]) so that (2) holds with any $J>1$. 
Next, we show in $₫ 6$ that there exists a quasiregular mapping $f$ on $\mathbb{R}^{2}$ with an essential singularity at infinity, such that the closure of $I(f)$ has a bounded component. Thus while the result of [16 that $I(f)$ has at least one unbounded component extends to quasiregular mappings by Theorem 1.1. Eremenko's theorem [5] that all components of the closure of $I(f)$ are unbounded does not.

We remark finally that it is easy to show that if $f$ is quasimeromorphic with infinitely many poles in $\mathbb{R}^{N}$, then $I(f)$ is nonempty, and for completeness we outline how this is proved in 97 , using the "jumping from pole to pole" method [3, 4].

\section{Theorems of Rickman and Miniowitz}

Let $G$ be a domain in $\mathbb{R}^{N}$. A continuous mapping $f: G \rightarrow \mathbb{R}^{N}$ is called quasiregular [15] if $f$ belongs to the Sobolev space $W_{N, l o c}^{1}(G)$ and there exists $K \in[1, \infty)$ such that $\left|f^{\prime}(x)\right|^{N} \leq K J_{f}$ a.e. in $G$. Moreover, $f$ is called $K$-quasiregular if its inner and outer dilatations do not exceed $K$ : for the details and equivalent definitions we refer the reader to [15]. Furthermore, if $f: G \rightarrow \overline{\mathbb{R}^{N}}=\mathbb{R}^{N} \cup\{\infty\}$ is continuous, then $f$ is called quasimeromorphic 9 if each $x \in G$ has a neighbourhood $U_{x}$ such that either $f$ or $g \circ f$ is a quasiregular map of $U_{x}$ into $\mathbb{R}^{N}$, where $g$ is a sense-preserving Möbius map of $\overline{\mathbb{R}^{N}}$ with $g(\infty) \in \mathbb{R}^{N}$.

Rickman proved [13, 15] that given $N \geq 2$ and $K \geq 1$ there exists an integer $C(N, K)$ such that if $f$ is $K$-quasiregular on $\mathbb{R}^{N}$ and omits $C(N, K)$ distinct values $a_{j} \in \mathbb{R}^{N}$, then $f$ is constant. Here $C(2, K)=2$ because a quasiregular mapping in $\mathbb{R}^{2}$ may be written as the composition of a quasiconformal mapping with an entire function, but for $N \geq 3$ this integer $C(N, K)$ in general exceeds 2 [14, 15].

Miniowitz [10] established for quasiregular mappings the following direct analogue of Zalcman's lemma [19, 20]. A family $F$ of $K$-quasiregular mappings on the unit ball $B^{N}$ of $\mathbb{R}^{N}$ is not normal if and only if there exist

$$
f_{n} \in F, \quad x_{n} \in B^{N}, \quad x_{n} \rightarrow \hat{x} \in B^{N}, \quad \rho_{n} \rightarrow 0+
$$

and a nonconstant $K$-quasiregular mapping $f: \mathbb{R}^{N} \rightarrow \mathbb{R}^{N}$ with the property that $f_{n}\left(x_{n}+\rho_{n} x\right) \rightarrow f(x)$ locally uniformly in $\mathbb{R}^{N}$, with respect to the the spherical distance $\chi(x, y)$ on $\mathbb{R}^{N}$. Using this she established the following analogue of Montel's theorem, in which $C(N, K)$ is the integer from Rickman's theorem [13.

Theorem 2.1 ([10]). Let $N \geq 2, K>1, \varepsilon>0$ and let $D$ be a domain in $\mathbb{R}^{N}$. Let $F$ be a family of functions $K$-quasiregular on $D$ with the following property. Each $f \in F$ omits $q=C(N, K)$ values $a_{1}(f), \ldots, a_{q}(f)$ on $D$, which may depend on $f$ but satisfy

$$
\chi\left(a_{j}(f), a_{k}(f)\right) \geq \varepsilon \quad \text { for } \quad j \neq k .
$$

Then $F$ is normal on $D$.

Theorem 2.1 leads at once to the following standard lemma of Schottky type.

Lemma 2.1. Let $N \geq 2$ and $K>1$. Then there exists $Q>2$ with the following property. Let $f$ be $K$-quasiregular on the set $\left\{x \in \mathbb{R}^{N}: 1<|x|<4\right\}$ such that $f$ omits $q=C(N, K)$ values $y_{1}, \ldots, y_{q}$, with

$$
\left|y_{j}\right|=4^{j-1}, \quad j=1, \ldots, q .
$$

If $\min \{|f(x)|:|x|=2\} \leq 2$, then $\max \{|f(x)|:|x|=2\} \leq Q$. 


\section{Two Lemmas neEded For Theorem 1.1}

We need the following two facts, the first of which is from Newman's book [1], Exercise, p. 84]:

Lemma 3.1. Let $G$ be a continuum in $\overline{\mathbb{R}^{N}}=\mathbb{R}^{N} \cup\{\infty\}$ such that $\infty \in G$, and let $H$ be a component of $\mathbb{R}^{N} \cap G$. Then $H$ is unbounded.

This leads to the second fact we need:

Lemma 3.2. Let $E$ be a continuum in $\overline{\mathbb{R}^{N}}$ such that $\infty \in E$, and let $g: \mathbb{R}^{N} \rightarrow \mathbb{R}^{N}$ be a continuous open mapping. Then the preimage

$$
g^{-1}(E)=\left\{x \in \mathbb{R}^{N}: g(x) \in E\right\}
$$

cannot have a bounded component.

For completeness we give a proof of Lemma 3.2 in $₫ 8$

\section{An analogue of Bohr's theorem}

Let $f: D_{R} \rightarrow \mathbb{R}^{N}$ be $K$-quasiregular, where $D_{R} \subseteq \mathbb{R}^{N}$ is a domain containing the set $B_{R}$ in (11), and assume that $f$ satisfies (2) for some $J>1$. For $0 \leq r<s \leq \infty$ set

$$
A(r, s)=\left\{x \in \mathbb{R}^{N}: r<|x|<s\right\}
$$

Using (2) choose $s_{0}>R$ such that

$$
M(r, f)>M(R, f) \text { for all } r \geq s_{0} .
$$

Then $M(r, f)$ is strictly increasing on $\left[s_{0}, \infty\right)$ because if $s_{0} \leq r_{1}<r_{2}<\infty$ and $M\left(r_{2}, f\right) \leq M\left(r_{1}, f\right)$, then $|f(x)|$ has a local maximum at some $\hat{x} \in A\left(R, r_{2}\right)$, which contradicts the fact that nonconstant quasiregular mappings send open sets to open sets [15, Theorem 4.1, p. 16]. Following Dominguez [4] we establish a lemma analogous to Bohr's theorem.

Lemma 4.1. Let $c=1 /(2 Q)$, where $Q$ is the constant of Lemma 2.1. Then for all sufficiently large $\rho$ there exists $L \geq c M(\rho / 2, f)$ such that

$$
S(0, L)=\left\{x \in \mathbb{R}^{N}:|x|=L\right\} \subseteq f(A(R, \rho)) .
$$

Proof. Using (2) let $\rho$ be so large that

$$
\rho>4 R \quad \text { and } \quad S=c M(\rho / 2, f)>2 T=4 M(R, f),
$$

and assume that the assertion of the lemma is false for $\rho$. Then for $j=1, \ldots, q$, where $q=C(N, K)$ is the integer from Rickman's Picard theorem [13] (see \$2), there exists $a_{j} \in \mathbb{R}^{N}$ with

$$
\left|a_{j}\right|=4^{j-1} S \quad \text { and } \quad a_{j} \notin f(A(R, \rho)) .
$$

Furthermore, there exists $x_{1} \in A(R, \rho / 2)$ such that $\left|f\left(x_{1}\right)\right|=S$. To see this join a point $x_{0}$ on $S(0, \rho / 2)$ such that $\left|f\left(x_{0}\right)\right|=M(\rho / 2, f)$ to $S(0, R)$ by a radial segment and use (4) and the fact that $c<1$. Let $G$ be the component of the set

$$
\left\{x \in \mathbb{R}^{N}: T<|f(x)|<2 S\right\}
$$

which contains $x_{1}$. Then $G \subseteq A(R, \infty)$ by (4). Suppose first that $G \subseteq A(R, \rho / 2)$. Then the closure $\bar{G}$ of $G$ lies in $A(R, \rho)$ by (4) again. Choose a geodesic $\sigma \subseteq S(0, S)$ joining $f\left(x_{1}\right)$ to $a_{1}$. Let

$$
\mu=\inf \left\{\left|f(x)-a_{1}\right|: x \in \bar{G}, f(x) \in \sigma\right\}
$$


THE ESCAPING SET OF A QUASIREGULAR MAPPING

and take $\zeta_{n} \in \bar{G}$ with $f\left(\zeta_{n}\right) \in \sigma$ and $\left|f\left(\zeta_{n}\right)-a_{1}\right| \rightarrow \mu$. Then we may assume that $\zeta_{n} \rightarrow \hat{\zeta} \in \bar{G}$, and we have $f(\hat{\zeta}) \in \sigma$ and so $\hat{\zeta} \in G$. But then the open mapping theorem forces $\mu=\left|f(\hat{\zeta})-a_{1}\right|=0$, which contradicts (5).

Thus $G \nsubseteq A(R, \rho / 2)$, and this implies, using (4) again, that there must exist $x_{2}$ on $S(0, \rho / 2)$ such that $\left|f\left(x_{2}\right)\right| \leq 2 S$. By (4) and (5) the function $g(x)=f(x \rho / 4) / S$ is $K$-quasiregular on $A(1,4)$ and omits the $q$ values $y_{j}=a_{j} / S$, which satisfy $\left|y_{j}\right|=$ $4^{j-1}$. Since $\left|g\left(4 x_{2} / \rho\right)\right| \leq 2$, Lemma 2.1 implies that $|g(x)| \leq Q$ for $|x|=2$, which gives

$$
M(\rho / 2, f) \leq Q S=Q c M(\rho / 2, f)=\frac{M(\rho / 2, f)}{2},
$$

a contradiction.

\section{Proof of Theorem 1.1}

Again let $f: D_{R} \rightarrow \mathbb{R}^{N}$ be $K$-quasiregular, where $D_{R} \subseteq \mathbb{R}^{N}$ is a domain containing the set $B_{R}$ in (11), but this time assume that $f$ satisfies (2) for some large positive $J$. Retain the notation of \&4. Following Dominguez' method [4] let $\rho_{0}>R$ be so large that every $\rho \geq \rho_{0}$ satisfies the conclusion of Lemma 4.1 and further that with the same constant $c$ as in Lemma 4.1.

$$
c M(\rho / 2, f)>4 \rho>\rho>M(R, f) \text { for all } \rho \geq \rho_{0},
$$

which is possible by (2) and the assumption that $J$ is large. Fix $\rho \geq \rho_{0}$.

Lemma 5.1. There exist bounded open sets $G_{0}, G_{1}, \ldots$ with the following properties.

(i) The set $\overline{\mathbb{R}^{N}} \backslash G_{n}$ has two components, namely

$$
\widetilde{G}_{n}=\overline{B(0, R)}=\left\{x \in \mathbb{R}^{N}:|x| \leq R\right\}
$$

and $G_{n}^{*}=A_{n}$, which satisfies $\infty \in A_{n}$.

(ii) We have

$$
\left\{x \in \mathbb{R}^{N}: R<|x| \leq 2^{n} \rho\right\} \subseteq G_{n} .
$$

(iii) The sets $G_{n}, A_{n}$ and $\gamma_{n}=\partial A_{n}$ satisfy

$$
\gamma_{n+1} \subseteq f\left(\gamma_{n}\right) \quad \text { and } \quad f\left(G_{n}\right) \cap A_{n+1}=\emptyset .
$$

Proof. The open sets $G_{n}$ will be constructed inductively. We begin by setting $G_{0}=A\left(R, \rho^{\prime}\right)$ for some $\rho^{\prime}>\rho$, so that (7) obviously is satisfied for $n=0$. It remains to show how to construct $G_{n+1}$ given the existence of $G_{0}, \ldots, G_{n}$ for some $n \geq 0$. The fact that $f$ maps open sets to open sets gives

$$
\partial f\left(G_{n}\right) \subseteq f\left(\partial G_{n}\right)=f(S(0, R)) \cup f\left(\gamma_{n}\right),
$$

using (i) and the definition $\gamma_{n}=\partial A_{n}$. By Lemma 4.1, (6) and (7) there exists

$$
T_{n} \geq c M\left(2^{n-1} \rho, f\right)>2^{n+2} \rho \text { with } \quad S\left(0, T_{n}\right) \subseteq f\left(A\left(R, 2^{n} \rho\right)\right) \subseteq f\left(G_{n}\right) .
$$

Now $f\left(G_{n}\right)$ is a bounded open set, so let $A_{n+1}$ be the component of $\overline{\mathbb{R}^{N}} \backslash f\left(G_{n}\right)$ which contains $\infty$ and set

$$
\gamma_{n+1}=\partial A_{n+1} .
$$

Then by (10) we have

$$
\gamma_{n+1} \subseteq A_{n+1} \subseteq A\left(2^{n+2} \rho, \infty\right),
$$


and (6), (9) and (11) imply the first assertion of (8). Let

$$
G_{n+1}=\mathbb{R}^{N} \backslash\left(\overline{B(0, R)} \cup A_{n+1}\right) .
$$

Then (i) is satisfied with $n$ replaced by $n+1$, and the second assertion of (8) follows from the definition of $A_{n+1}$. Finally (12) shows that (7) is satisfied with $n$ replaced by $n+1$, and so the induction is complete.

Lemma 5.2. Let $w \in \gamma_{n}$. Then there exists $z_{n} \in \gamma_{0}$ with $f^{n}\left(z_{n}\right)=w$ and

$$
f^{m}\left(z_{n}\right) \in \gamma_{m} \quad \text { for } \quad m=0, \ldots, n \text {. }
$$

Proof. This is easily proved using induction and (8).

Now take a sequence of points $z_{n} \in \gamma_{0}$ satisfying (13). We may assume that $\left(z_{n}\right)$ converges to $\hat{z} \in \gamma_{0}$, and we have, by (13),

$$
f^{m}(\hat{z})=\lim _{n \rightarrow \infty} f^{m}\left(z_{n}\right) \in \gamma_{m} \quad \text { for each } m \geq 0 .
$$

Using (12) we get $\hat{z} \in I(f)$, and hence $I(f)$ is nonempty. This proves the first assertion of Theorem 1.1.

The second assertion will be established by modifying the method of Rippon and Stallard [16, so assume that $f$ is $K$-quasiregular in $\mathbb{R}^{N}$ and take $\hat{z}$ satisfying (14). As before let $A_{n}=G_{n}^{*}$ be the component of $\overline{\mathbb{R}^{N}} \backslash G_{n}$ containing $\infty$, and let $L_{n}$ be the component of $f^{-n}\left(A_{n}\right)$ containing $\hat{z}$, which is well-defined since $f^{n}(\hat{z}) \in \gamma_{n}$ and $\gamma_{n}=\partial A_{n}$ by definition.

Lemma 5.3. $L_{n}$ is closed and unbounded.

Proof. $L_{n}$ is closed since $A_{n}$ is closed, and $L_{n}$ is unbounded by Lemma 3.2

Lemma 5.4. We have $L_{n+1} \subseteq L_{n}$ for $n=0,1, \ldots$

Proof. Suppose that $f^{n+1}\left(z^{\prime}\right) \in A_{n+1}$ but $f^{n}\left(z^{\prime}\right) \notin A_{n}$. Thus either $\left|f^{n}\left(z^{\prime}\right)\right| \leq R$ or $f^{n}\left(z^{\prime}\right) \in G_{n}$, from which we obtain $f^{n+1}\left(z^{\prime}\right) \notin A_{n+1}$, in the first case from (6) and (17) and in the second case from (8), and this is a contradiction. Hence if $z^{\prime} \in L_{n+1}$, then $z^{\prime}$ lies in a component of $f^{-n-1}\left(A_{n+1}\right)$ which contains $\hat{z}$, and this component in turn lies in a component of $f^{-n}\left(A_{n}\right)$. Hence we get $z^{\prime} \in L_{n}$.

We may now write

$$
K_{n}=L_{n} \cup\{\infty\}, \quad\{\hat{z}, \infty\} \subseteq K_{n+1} \subseteq K_{n}, \quad\{\hat{z}, \infty\} \subseteq K=\bigcap_{n=0}^{\infty} K_{n} .
$$

Since $K_{n}$ is compact and connected so is $K$ [11, Theorem 5.3, p. 81]. Let $\Gamma$ be the component of $K \backslash\{\infty\}$ which contains $\hat{z}$. Then $\Gamma$ is unbounded by Lemma 3.1. Now for $w \in \Gamma$ we have $w \in L_{n}$ and so $f^{n}(w) \in A_{n}=G_{n}^{*}$, so that $w \in I(f)$ by (7). This completes the proof of Theorem 1.1

We do not know whether the second conclusion of Theorem 1.1 holds if $f$ is only quasiregular on the set $B_{R}$ in (1), but this seems unlikely. The difficulty is that for large $n$ we cannot control the behaviour of $f^{n}$ near $S(0, R)$ and so the component $L_{n}$ in Lemma 5.3 may in principle be bounded. 


\section{A QUASIREgUlar MAPPING $f$ FOR WHICH $\overline{I(f)}$ HAS A BOUNDED COMPONENT}

To show that there exists a quasiregular mapping $f: \mathbb{C} \rightarrow \mathbb{C}$ such that the closure of the escaping set $I(f)$ has a bounded component, we begin by constructing a quasiconformal map $g$ with the following properties. For each $z$ in the punctured $\operatorname{disc} A:=\{z \in \mathbb{C}: 0<|z|<1\}$ the iterates $g^{n}$ satisfy $\lim _{n \rightarrow \infty}\left|g^{n}(z)\right|=1$, and we have $\lim _{n \rightarrow \infty} g^{n}(1 / 2)=1$. On the other hand there exist annuli $A_{n} \subseteq A$ such that $g$ maps $A_{n}$ onto $A_{n+1}$, but with sufficient rotation that for each $z \in A_{n}$ infinitely many of the forward images $g^{k}(z)$ lie away from 1. A map $h$ is then obtained from $g$ by conjugation with a Möbius map $L$ which sends 1 to $\infty$, and finally $h$ is interpolated on a sector to ensure that the resulting function has an essential singularity at infinity.

We will use the fact that if $p$ is quasiregular on a domain $D \subseteq \mathbb{C}$ and

$$
p_{z}=\frac{\partial p}{\partial z}=\frac{1}{2}\left(\frac{\partial p}{\partial x}-i \frac{\partial p}{\partial y}\right)
$$

is bounded below in modulus on $D$, and if $q$ is continuous and such that the partial derivatives $q_{x}, q_{y}$ are sufficiently small on $D$, then $p+q$ is quasiregular on $D$. If $0 \notin D \cup p(D)$ the same property may be applied locally to $\log p$ as a function of $\log z$.

Turning to the detailed construction, we define $a:[1,2] \rightarrow[0, \pi / 4]$ by

$$
a(r)=\frac{\pi}{4}-\arcsin \left(\frac{\sqrt{2}}{2 r}\right) .
$$

Then an application of the sine rule shows that the line segment

$$
\operatorname{Re} z=1+\operatorname{Im} z, \quad 1 \leq|z| \leq 2,
$$

is parametrized by $z=r e^{i a(r)}$.

For $c>0$ we define $g: \mathbb{C} \rightarrow \mathbb{C}$ as follows. Let $g(0)=0$ and for $z=r e^{i t}$ with $r>0$ and $-\pi \leq t \leq \pi$ set:

$$
g(z)= \begin{cases}\frac{4}{3} r \exp (i(t+c|\sin t|)), & 0<r<\frac{1}{2} ; \\ \frac{1}{2-r} \exp \left(i\left(t+c|\sin t|+c(1-r)^{2}\left|\sin \left(\frac{\pi}{1-r}\right)\right|\right)\right), & \frac{1}{2} \leq r<1 ; \\ r \exp \left(i\left(t+c(2-r) \sin \left(\frac{|t|-a(r)}{\pi-a(r)} \pi\right)\right)\right), & 1 \leq r \leq 2, a(r)<|t| ; \\ r \exp (i t), & 1 \leq r \leq 2,|t| \leq a(r) ; \\ r \exp (i t), & r>2 .\end{cases}
$$

Then $g$ is continuous on $\mathbb{C}$. Moreover, if $c$ is sufficiently small, then $g$ is quasiconformal, and in particular we choose $c<\pi / 4$. Note that, by the choice of $a(r)$,

$$
g(z)=z \quad \text { if } \quad \operatorname{Re} z \geq|\operatorname{Im} z|+1 .
$$

For $n \in \mathbb{N}$ we have

$$
g\left(1-\frac{1}{n+1}\right)=1-\frac{1}{n+2}
$$


For $n \in \mathbb{N}, n \geq 2$, we consider the annulus

$$
A_{n}:=\left\{z \in \mathbb{C}: 1-\frac{1}{n+1 / 4}<|z|<1-\frac{1}{n+3 / 4}\right\} \text {. }
$$

Then $g\left(A_{n}\right)=A_{n+1}$.

Lemma 6.1. For each $z \in A_{n}$ with $\operatorname{Re} z>0$ there exists $k \in \mathbb{N}$ with $\operatorname{Re} g^{k}(z) \leq 0$.

Proof. Let $z \in A_{m}$ and suppose first that $0<t:=\arg z<\pi / 2$. Then

$$
\pi>t+\frac{\pi}{2}>t+2 c \geq \arg g(z) \geq t+c \sin t \geq t+\frac{2 c}{\pi} t=\left(1+\frac{2 c}{\pi}\right) t .
$$

On the other hand, if $-\pi / 2<t=\arg z \leq 0$, then

$$
\frac{\pi}{2}>\arg g(z) \geq t+c|\sin t|+\frac{c \sqrt{2}}{2(m+3 / 4)^{2}} \geq\left(1-\frac{2 c}{\pi}\right) t+\frac{c^{\prime}}{(m+1)^{2}}>-\frac{\pi}{2},
$$

where $c^{\prime}:=\frac{1}{2} c \sqrt{2}$. In particular, (17) and (18) both hold with $\arg g(z)$ the principal argument.

Suppose then that there exists $z \in A_{n}$ with $\operatorname{Re} g^{k}(z)>0$ for all integers $k \geq 0$, and set $t_{k}=\arg g^{k}(z) \in(-\pi / 2, \pi / 2)$. Then $g^{k}(z) \in A_{n+k}$. If there exists $k \geq 0$ with $0<t_{k}<\pi / 2$, then by repeated application of (17) we obtain $k^{\prime}>k$ with $t_{k^{\prime}} \in(\pi / 2, \pi)$, a contradiction. Hence we must have $-\pi / 2<t_{k} \leq 0$ for all $k \geq 0$. But then repeated application of (18) gives, for large $k$,

$$
t_{k-1} \geq\left(1-\frac{2 c}{\pi}\right)^{k-1} t_{0}, \quad t_{k} \geq\left(1-\frac{2 c}{\pi}\right) t_{k-1}+\frac{c^{\prime}}{(n+k)^{2}}>0,
$$

again a contradiction.

With the Möbius transformation

$$
L(z)=\frac{1}{1-z}
$$

we now consider the map $h:=L \circ g \circ L^{-1}$. Then $h$ is a quasiconformal self-map of the plane. Moreover, (15) gives $h(z)=z$ if $\operatorname{Re} L^{-1}(z) \geq\left|\operatorname{Im} L^{-1}(z)\right|+1$, which is equivalent to $\operatorname{Re} z \leq-|\operatorname{Im} z|$, and we have

$$
L\left(A_{n}\right) \subseteq\{z \in \mathbb{C}: \operatorname{Re} z>0\} \text { and } h\left(L\left(A_{n}\right)\right)=L\left(A_{n+1}\right),
$$

using the fact that $g\left(A_{n}\right)=A_{n+1}$.

It follows from (16) that

$$
h(n+1)=n+2 \quad \text { for } \quad n \in \mathbb{N},
$$

and we deduce at once that $2 \in I(h)$. Next we show that $L\left(A_{n}\right) \cap I(h)=\emptyset$ for every integer $n \geq 2$. In fact, suppose that $n \geq 2$ and $u \in L\left(A_{n}\right) \cap I(h)$. Then there exists $j_{0} \in \mathbb{N}$ such that $\left|h^{j}(u)\right|>1$ for $j \geq j_{0}$. Put $w:=h^{j_{0}}(u)$ and $m:=n+j_{0}$. Then $L^{-1}(w) \in A_{m}$ by (19), and Lemma 6.1 gives $k \geq 0$ with $\operatorname{Re} g^{k}\left(L^{-1}(w)\right) \leq 0$. Since $|L(z)| \leq 1$ for $\operatorname{Re} z \leq 0$ we deduce that

$$
\left|h^{k+j_{0}}(u)\right|=\left|h^{k}(w)\right|=\left|L\left(g^{k}\left(L^{-1}(w)\right)\right)\right| \leq 1,
$$

contradicting the choice of $j_{0}$. Thus $L\left(A_{n}\right) \cap I(h)=\emptyset$.

Since $A_{2}$ separates $\frac{1}{2}$ from 1 it follows that 2 lies in the bounded component of the complement of $L\left(A_{2}\right)$, and we deduce that the component of $\overline{I(h)}$ containing 2 is bounded. 
To construct a quasiregular map $f: \mathbb{C} \rightarrow \mathbb{C}$ with an essential singularity at $\infty$ for which the closure of $I(f)$ has a bounded component, we put $f(z)=h(z)$ for $\operatorname{Re} z \geq-|\operatorname{Im} z|$ and $f(z)=z+d \exp \left(z^{4}\right)$ for $\operatorname{Re} z \leq-|\operatorname{Im} z|-1$, where $d$ is a small positive constant. In the remaining region $\Omega$ we define $f$ by interpolation, using

$f(z)=z-d \phi(z), \quad \phi(z)=(\operatorname{Re} z+|\operatorname{Im} z|) \exp \left(z^{4}\right) \quad$ for $\quad-1<\operatorname{Re} z+|\operatorname{Im} z|<0$.

Since $\exp \left(z^{4}\right)$ tends to 0 rapidly as $z$ tends to infinity in $\Omega$, it is then clear that the partial derivatives of $\phi$ are bounded on $\Omega$, so that $f$ is quasiregular on $\Omega$ because $d$ is small.

In particular we have $f(z)=h(z)$ for $\operatorname{Re} z>0$, and so it follows from (20) that $2 \in I(f)$, whereas $L\left(A_{n}\right) \cap I(f)$ is again empty using (19). Thus the component of $\overline{I(f)}$ containing 2 is bounded.

\section{The QUASIMEROMORPHIC CASE}

Let $f$ be nonconstant and $K$-quasimeromorphic on the set $B_{R}$ defined in (11), with a sequence of poles tending to $\infty$, and set $R_{-1}=R$. Choose $x_{j}, D_{j}, R_{j}$ for $j=0,1,2, \ldots$ as follows. Each $x_{j}$ is a pole of $f$, and $D_{j}$ is a bounded component of the set $\left\{x \in B_{R}: R_{j}<|f(x)| \leq \infty\right\}$ which contains $x_{j}$ but no other pole of $f$, such that $D_{j}$ is mapped by $f$ onto $\left\{y \in \overline{\mathbb{R}^{N}}: R_{j}<|y| \leq \infty\right\}$. Moreover, by choosing $R_{j+1}$ and $x_{j+1}$ sufficiently large, we may ensure that

$$
\left|x_{j+1}\right|>4 R_{j} \quad \text { and } \quad D_{j+1} \subseteq\left\{x \in \mathbb{R}^{N}: 2 R_{j}<|x|<\infty\right\} \quad \text { for } \quad j \geq-1 .
$$

Since $|f(x)|=R_{j}$ for all $x \in \partial D_{j}$ we may write, for $j \geq 0$, using (21),

$$
C_{j}=\left\{x \in D_{j}: f(x) \in D_{j+1}\right\} \subseteq \overline{C_{j}} \subseteq D_{j} .
$$

Now set

$$
X_{0}=\overline{C_{0}}, \quad X_{j+1}=\left\{x \in X_{j}: f^{j+1}(x) \in \overline{C_{j+1}}\right\} .
$$

Evidently $X_{0}$ is compact. Assuming that $X_{j}$ is compact, it then follows that $X_{j+1}$ is the intersection of a compact set with the closed set $f^{-j-1}\left(\overline{C_{j+1}}\right)$ and so is compact. Hence the $X_{j}$ form a nested sequence of compact sets. We assert that

$$
f^{j}\left(X_{j}\right)=\overline{C_{j}} .
$$

We clearly have $f^{j}\left(X_{j}\right) \subseteq \overline{C_{j}}$ by (23), and (24) is obviously true for $j=0$, so assume the assertion for some $j \geq 0$ and take $w \in \overline{C_{j+1}}$. Since $f$ maps $D_{j}$ onto $\left\{y \in \overline{\mathbb{R}^{N}}: R_{j}<|y| \leq \infty\right\}$, it follows from (21) and (22) that there exists $v \in C_{j}$ with $f(v)=w$. Hence there exists $x \in X_{j}$ with $f^{j}(x)=v$ and $f^{j+1}(x)=w$, completing the induction.

Again since $f$ maps $D_{j}$ onto $\left\{y \in \overline{\mathbb{R}^{N}}: R_{j}<|y| \leq \infty\right\}$, we evidently have $C_{j} \neq \emptyset$ and so $X_{j}$ is nonempty by (24). Hence there exists $x$ lying in the intersection of the $X_{j}$, so that $f^{j}(x) \in \overline{C_{j}}$ and $x \in I(f)$ by (21) and (22).

\section{Proof of Lemma 3.2}

To establish Lemma 3.2 let $E$ and $g$ be as in the statement and assume that $g^{-1}(E)$ is nonempty since otherwise there is nothing to prove. Note first that $g^{-1}(E)$ is a closed subset of $\mathbb{R}^{N}$ by continuity. Thus

$$
F=g^{-1}(E) \cup\{\infty\}
$$


is a compact subset of $\overline{\mathbb{R}^{N}}$. In order to prove Lemma 3.2 it therefore suffices in view of Lemma 3.1 to show that $F$ is connected. Suppose that this is not the case. Then there is a partition of $F$ into nonempty disjoint relatively closed (and so closed) sets $H_{1}, H_{2}$ such that $\infty \in H_{2}$. Let $W=\mathbb{R}^{N} \backslash H_{2}$. Then $W$ is an open subset of $\mathbb{R}^{N}$, and $g\left(W \backslash H_{1}\right) \cap E=\emptyset$. Moreover, $H_{1}$ is a closed subset of $\overline{\mathbb{R}^{N}}$ and thus compact, and hence a compact subset of $\mathbb{R}^{N}$ since $\infty \in H_{2}$. Thus $g\left(H_{1}\right)$ is compact and thus a nonempty closed subset of $E$.

Now suppose that there exist $y_{n} \in E \backslash g\left(H_{1}\right)$ with $y_{n} \rightarrow \widetilde{y} \notin E \backslash g\left(H_{1}\right)$. Since $E$ is compact we have $\widetilde{y} \in E$ and so $\widetilde{y} \in g\left(H_{1}\right)$. Hence there exists $\widetilde{x} \in H_{1}$ with $g(\widetilde{x})=\widetilde{y}$ and for large enough $n$ there exists $x_{n}$ close to $\widetilde{x}$ with $g\left(x_{n}\right)=y_{n} \in E \backslash g\left(H_{1}\right)$. But then we must have $x_{n} \in H_{1}$, since $g\left(W \backslash H_{1}\right) \cap E=\emptyset$, and this is a contradiction. So $E \backslash g\left(H_{1}\right)$ is also closed, but evidently nonempty since $g\left(\mathbb{R}^{N}\right) \subseteq \mathbb{R}^{N}$ and $\infty \in E$, which contradicts the hypothesis that $E$ is connected.

\section{REFERENCES}

[1] W. Bergweiler, Iteration of meromorphic functions, Bull. Amer. Math. Soc. 29 (1993), 151188. MR:1216719 (94c:30033)

[2] W. Bergweiler, Fixed points of composite entire and quasiregular maps, Ann. Acad. Sci. Fenn. Math. 31 (2006), 523-540. MR.2248829 (2007g:30043)

[3] W. Bergweiler, P.J. Rippon and G.M. Stallard, Dynamics of meromorphic functions with direct or logarithmic singularities, Proc. London Math. Soc. 2008; doi: 10.1112/plms/pdn007.

[4] P. Dominguez, Dynamics of transcendental meromorphic functions, Ann. Acad. Sci. Fenn. Math. 23 (1998), 225-250. MR1601879 (99b:30031)

[5] A. Eremenko, On the iteration of entire functions, Dynamical Systems and Ergodic Theory, Banach Center Publ. 23, Polish Scientific Publishers, Warsaw, 1989, 339-345. MR1102727 (92c:30027)

[6] W.K. Hayman, The local growth of power series: A survey of the Wiman-Valiron method, Canad. Math. Bull. 17 (1974), 317-358. MR0385095(52:5965)

[7] A. Hinkkanen, G.J. Martin and V. Mayer, Local dynamics of uniformly quasiregular mappings, Math. Scand. 95 (2004), 80-100. MR2091483 (2005f:37094)

[8] T. Iwaniec and G. Martin, Geometric function theory and non-linear analysis, Clarendon Press, Oxford University Press, New York, 2001. MR,1859913 (2003c:30001)

[9] O. Martio, S. Rickman and J. Väisälä, Distortion and singularities of quasiregular mappings, Ann. Acad. Sci. Fenn. Ser. A I 465 (1970), 1-13. MR0267093 (42:1995)

[10] R. Miniowitz, Normal families of quasimeromorphic mappings, Proc. Amer. Math. Soc. 84 (1982), 35-43. MR633273 (83c:30026)

[11] M.H.A. Newman, Elements of the topology of plane sets of points, Cambridge, At the University Press, 1951. MR0044820(13:483a)

[12] L. Rempe, On a question of Eremenko concerning escaping components of entire functions, Bull. London Math. Soc. 39 (2007), 661-666. MR2346947

[13] S. Rickman, On the number of omitted values of entire quasiregular mappings, J. Analyse Math. 37 (1980), 100-117. MR583633 (81m:30030)

[14] S. Rickman, The analogue of Picard's theorem for quasiregular mappings in dimension three, Acta Math. 154 (1985), 195-242. MR781587 (86h:30039)

[15] S. Rickman, Quasiregular mappings, Ergebnisse der Mathematik und ihrer Grenzgebiete 26, Springer-Verlag, Berlin, 1993. MR.1238941 (95g:30026)

[16] P.J. Rippon and G.M. Stallard, On questions of Fatou and Eremenko, Proc. Amer. Math. Soc. 133 (2005), 1119-1126. MR2117213 (2005j:37069)

[17] H. Siebert, Fixed points and normal families of quasiregular mappings, J. Analyse Math. 98 (2006), 145-168. MR2254483 (2007e:30023)

[18] N. Steinmetz, Rational iteration, de Gruyter Studies in Mathematics 16, Walter de Gruyter, Berlin-New York, 1993. MR1224235 (94h:30035) 
[19] L. Zalcman, A heuristic principle in complex function theory, Amer. Math. Monthly 82 (1975), 813-817. MR0379852 (52:757)

[20] L. Zalcman, Normal families: New perspectives, Bull. Amer. Math. Soc. (N.S.) 35 (1998), 215-230. MR.1624862 (99g:30048)

Mathematisches Seminar, Christian-Albrechts-Universität ZU Kiel, Ludewig-MeynStr. 4, D-24098 Kiel, Germany

E-mail address: bergweiler@math.uni-kiel.de

School of Mathematical Sciences, University of Nottingham, Nottingham NG7 2RD, UNITED KingDOM

E-mail address: alastair.fletcher@nottingham.ac.uk

School of Mathematical Sciences, University of Nottingham, Nottingham NG7 2RD, UNITED KINGDOM

E-mail address: jkl@maths.nott.ac.uk

School of Mathematical Sciences, University of Nottingham, Nottingham NG7 2RD, United KingDOM

E-mail address: janis.meyer@maths.nottingham.ac.uk 